\title{
OPEN Creation of X-linked Alport syndrome rat model with Col4a5 deficiency
}

\author{
Masumi Namba ${ }^{1}$, Tomoe Kobayashi, ${ }^{1,5}$, Mayumi Kohno ${ }^{1,5}$, Takayuki Koyano ${ }^{1}$, Takuo Hirose ${ }^{2,3}$, \\ Masaki Fukushima ${ }^{1,4}$ \& Makoto Matsuyama ${ }^{1 凶}$
}

\begin{abstract}
Alport syndrome is an inherited chronic human kidney disease, characterized by glomerular basement membrane abnormalities. This disease is caused by mutations in COL4A3, COL4A4, or COL4A5 gene. The knockout mice for $\mathrm{Col} 4 \alpha 3, \mathrm{Col} 4 \alpha 4$, and $\operatorname{Col} 4 \alpha 5$ are developed and well characterized for the study of Alport syndrome. However, disease progression and effects of pharmacological therapy depend on the genetic variability. This model was reliable only to mouse. In this study, we created a novel Alport syndrome rat model utilizing the rGONAD technology, which generated rat with a deletion of the Col4 $\alpha 5$ gene. Col4 $\alpha 5$ deficient rats showed hematuria, proteinuria, high levels of BUN, Cre, and then died at 18 to 28 weeks of age (Hemizygous mutant males). Histological and ultrastructural analyses displayed the abnormalities including parietal cell hyperplasia, mesangial sclerosis, and interstitial fibrosis. Then, we demonstrated that $\alpha 3 / \alpha 4 / \alpha 5$ (IV) and $\alpha 5 / \alpha 5 / \alpha 6$ (IV) chains of type IV collagen disrupted in Col $4 \alpha 5$ deficient rats. Thus, Col $4 \alpha 5$ mutant rat is a reliable candidate for the Alport syndrome model for underlying the mechanism of kidney diseases and further identifying potential therapeutic targets for human renal diseases.
\end{abstract}

Type IV collagen networks are the structural foundation for all basement membranes, consist of 6 different chains from COL4A 1 to COL4A6 in mammals ${ }^{1,2}$. The 6 chains assemble into 3 types of heterotrimers (protomers) which have distinct tissue distribution and function. In the mammalian kidney, the glomerular basement membrane (GBM) requiring long-term maintenance of the glomerular filtration functions, is composed of $\alpha 3 / \alpha 4 / \alpha 5$ (IV) isoforms. Whereas a1/a1/a2 (IV) isoforms are found in all basement membranes, and a5/a5/a6 (IV) isoforms are detected in Bowman's capsule $(\mathrm{BC})^{3}$.

Alport syndrome is a basement membrane disorder, characterized by hereditary nephropathy that results in irreversible, progressive renal failure ${ }^{4}$. Alport syndrome is caused by mutations in COL4A3, COL4A4, or COL4A5 gene encoding type IV collagen $\alpha 3$, $\alpha 4$, or $a 5$ chains ${ }^{5}$. Defects in the COL4A5 gene cause X-linked Alport syndrome, which accounts for about $80 \%$ of Alport syndrome ${ }^{6}$. And the remaining cases are associated with mutations in COL4A3 or COL4A4 genes. The pathological events of Alport syndrome are very similar, are found that lack of $C O L 4 A 3, C O L 4 A 4$, or COL4A5 resulted in the disorganization of $\alpha 3 / \alpha 4 / \alpha 5$ (IV) network ${ }^{1}$.

In the past decades, several Alport syndrome animal models have been produced in mouse. The mutant mice for Col4a3, Col4a4, and Col4a 5 are developed and well characterized ${ }^{7-12}$. These model mice led important aspects of the renal disease. Whereas, there are some significant differences depend on genetic background in Alport syndrome model mice ${ }^{9,13,14}$. For example, $129 \mathrm{X} 1 / \mathrm{Sv}$ or C57BL/6 was different patterns of disease progression in $\mathrm{Col}_{4 \mathrm{a} 3^{-/}}$mice ${ }^{15}$. Indeed, in the human patients, the disease progression and effects of pharmacological therapy were shown to genetic variability ${ }^{6}$. However, in spite of variabilities displayed in the genetic background, only a few mammal models (only mice and dogs ${ }^{16}$ ) have been established for Alport syndrome.

The laboratory rat (Rattus norvegicus) is a common experimental model for the human diseases and the drug testing ${ }^{17-19}$. For example, Wistar Kyoto (WKY) rat strain is known to be uniquely susceptible to crescentic glomerulonephritis among the strains tested ${ }^{20,21}$. Injection of isologous monoclonal antibodies caused antiglomerular basement membrane antibody-induced glomerulonephritis (anti-GBM nephritis) in WKY rats ${ }^{22,23}$. Although there are several advantages comparing with mice, production of genetically engineered rat models has not yet been extensively proceeded during the past decades. The recent CRISPR/Cas9 system is the simplest

${ }^{1}$ Division of Molecular Genetics, Shigei Medical Research Institute, 2117 Yamada, Minami-ku, Okayama 701-0202, Japan. ${ }^{2}$ Division of Nephrology and Endocrinology, Faculty of Medicine, Tohoku Medical and Pharmaceutical University, Sendai, Japan. ${ }^{3}$ Department of Endocrinology and Applied Medicine, Tohoku University Graduate School of Medicine, Sendai, Japan. ${ }^{4}$ Shigei Medical Research Hospital, Okayama, Japan. ${ }^{5}$ These authors contributed equally: Tomoe Kobayashi and Mayumi Kohno. ${ }^{\bowtie}$ email: matsuyama@shigei.or.jp 
for generating rats carrying a modified genome $\mathrm{e}^{24,25}$, however, the standard method for genome-editing in mammals involves 3 major steps: isolation of zygotes from females, micromanipulation ex vivo, and transfer into pseudopregnant females. These 3 steps require extremely high level of technical expertise and its proficiency of the researchers as well as technicians. To simplify these complexed and laborious processes, we established to produce knock-out and knock-in mouse and rat with high efficiency, named as $i$-GONAD (mouse) and rGONAD (rat Genome-editing via Oviductal Nucleic Acids Delivery) ${ }^{26-30}$. The rGONAD method involves 2 key steps: an injection of the solution containing Cas9 protein, guide RNA and single strand DNA (ssDNA) into the oviduct, followed by electroporation. The rats that have undergone rGONAD are bred in routine way until birth. Moreover, rGONAD is highly efficient in both knock-out and knock-in rats ${ }^{28,30}$.

In the present study, we attempted to create Alport syndrome rat model using the rGONAD technology. We developed Col4a5 deficient mutant rats, identical to the Col4a5 G5X mutant mice ${ }^{10}$, which showed progressive glomerular disease and CKD phenotypes with renal fibrosis. And we investigated whether the mutant rats were applicable for the Alport syndrome model.

\section{Results}

Generation of Col4 $\alpha 5$ deficient rats by the rGONAD method. On the basis of human mutations as described previously, we introduced novel Col $4 \alpha 5$ deficient rats with CRISPR/Cas 9 and the rGONAD technology $\mathrm{y}^{28,30}$. Tandem STOP codons were integrated into 27 bases after the first ATG in the rat Col4 $\alpha 5$ gene (Supplementary Fig. 1a). The mutation was verified by PCR followed by DNA sequencing, resulting to be expressed only 9 amino acids of COL4A 5 at its N-terminus (Supplementary Fig. 1b,c). We detected no COL4A5 protein expression in Col4 $\alpha 5$ deficient male rats by immunofluorescence and Western blot analyses with monoclonal antibodies against the NC1 (C-terminus) domains of type IV collagens (See below; Figs. 7,8). In addition, the mutant rats which were integrated the other flame-shift tandem STOP codons (Col4a5 15 aa stop; Supplementary Fig. 2) and were deleted 56 bp including the first ATG (Col4a5 56 bp deletion; Supplementary Fig. 3), also generated, and revealed the same phenotype as Alport syndrome rats (Supplementary Fig. 2, 3). These data collectively indicate the successful generation of Col4a5 deficient rats.

Physiological analyses in Col4 $\alpha 5$ mutants. The following data are from the mutants rats shown in Supplementary Fig. 1a,b. Col4a5 deficient rats were viable, fertile, and expected Mendelian ratios. However, all hemizygous mutant males died from 18 to 28 weeks of age $(n=58$; Fig. 1a). Heterozygous mutant females died at 35 to 100 weeks of age, among them, approximately $30 \%$ of females survived over 100 weeks $(n=33$, Fig. 1a). To assess of functional and histological abnormalities of the kidney, we measured the hematuria and proteinuria in Col4a5 deficient rats. Hematuria was found from postnatal 21 days in hemizygous mutant males, and from 4 weeks of age in heterozygous mutant females (data not shown). Proteinuria was observed (about $6.0 \mathrm{mg} / 16 \mathrm{~h}$ ) by 6 weeks of age in hemizygous mutant males (see Supplementary Table 1), then, increased $>20.0 \mathrm{mg} / 16 \mathrm{~h}$ in all of mutant males after 12 weeks of age $(n=35)$, and $62 \%$ of mutant females in 16 week of age $(n=28 / 45)$ (Fig. 1b). On the contrary, these phenomena were not detected in wildtype male and female rats in any week of age (Fig. 1b).

The mutant males showed decrease of body weight and increase of urine volume, compared with those of wildtype males (Fig. 1c,d). The levels of blood urea nitrogen (BUN) and creatinine (Cre) in serum highly increased in 20 weeks of age of mutant males (Fig. 1e,f). The protein/creatinine ratio was elevated in mutant males from 8 weeks of age, and creatinine clearance ratio was significantly lower in mutant males by 20 weeks of age (Fig. 1g,h).

Renal histology in Col4 $\alpha 5$ deficient rats. The kidney sections from wildtype and Col4a5 deficient rats were stained with hematoxylin and eosin (HE), periodic acid Schiff (PAS), periodic acid methenamine silver (PAM), and Masson trichrome (MT) (Fig. 2). At 8 weeks of age, the glomeruli exhibited capillary tuft collapse in hemizygous mutant males (Fig. 2a). However, the kidney displayed overall sparing of the tubulointerstitium (Fig. 2a). By the 20 weeks of age in hemizygous mutant males, substantial numbers of glomeruli revealed the abnormalities, including parietal cell hyperplasia mimicking crescent formation, focal sclerosis, and with overall sparing of the tubulointerstitium (Fig. 2b, Supplementary Fig. 4). The kidney sections from heterozygous mutant females at 8-20 weeks of age displayed focal abnormalities of the glomeruli and tubulointerstitium (Fig. 2a,b, Supplementary Fig. 4).

To analyze the 3 dimensional ultrastructure of GBM, low-vacuum scanning electron microscopy (LVSEM) was performed, as described previously ${ }^{31}$. LVSEM revealed the coarse meshwork structure of the GBM with numerous pin-holes in hemizygous mutant males, in contrast to the smoothly arranged surface in wildtype males (Fig. 3, Supplementary Fig. 5). The transmission electron microscopy (TEM) image of the GBM in mutant males exhibited partial thickening from 8 weeks of age, and thickening of the GBM became accentuated and widely spread with increasing age (Fig. 3, Supplementary Fig. 5). Moreover, TEM also revealed substantial thinning of the GBM and split or fragmentation of the lamina densa by 20 weeks of age (Fig. 3, Supplementary Fig. 5).

Renal glomerular and tubulointerstitial fibrosis in Col4 $\alpha \mathbf{5}$ deficient rats. To evaluate fibrosis of the glomeruli and tubulointerstitium in Col4a5 deficient rats, we examined kidney sections from wildtype and Col4a 5 deficient rats stained with antibodies specific for $\alpha$-smooth muscle actin ( $\alpha$-SMA) and fibronectin (Fig. 4). Immunostaining showed that expression level of $\alpha$-SMA, a maker of renal glomerular and tubulointerstitial fibrosis, was increased around the glomeruli from hemizygous mutant males when compared with those of wildtype littermates at 20 weeks of age, but it was not detected in the renal tubular epithelia (Fig. $4 a$ ). The a-SMA expression of kidney sections from the heterozygous females was rarely detectable at 20 weeks of age 
a
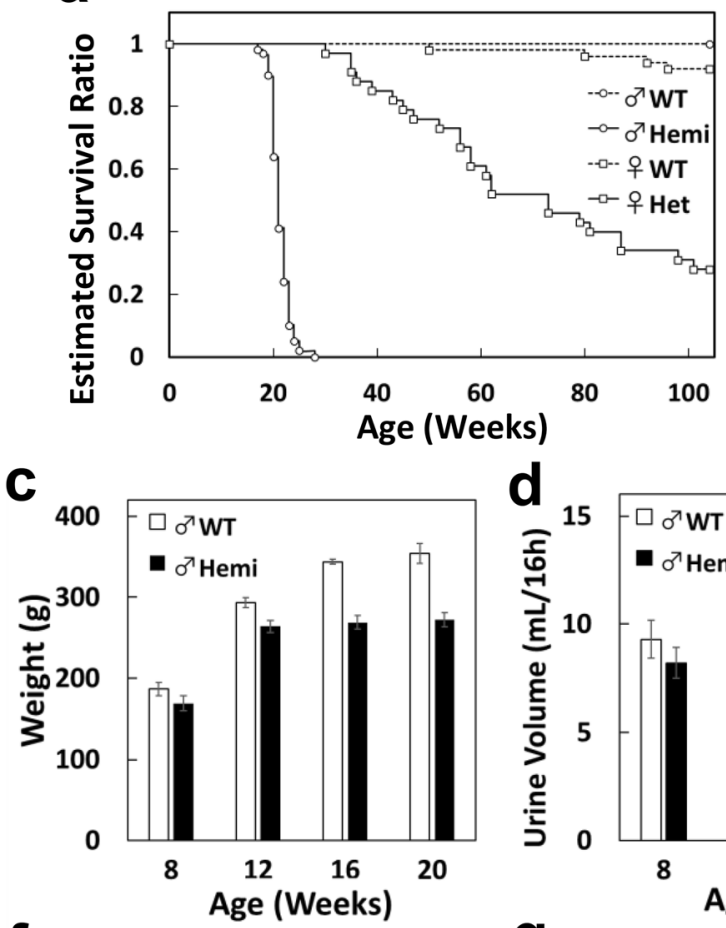

f

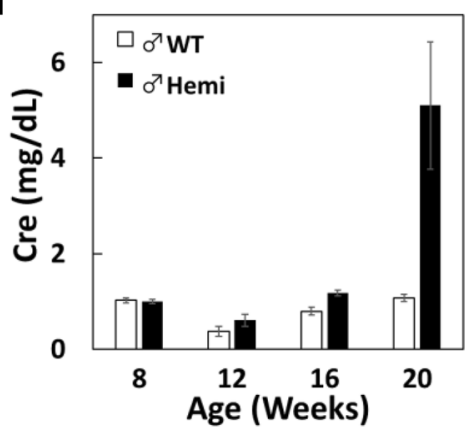

b

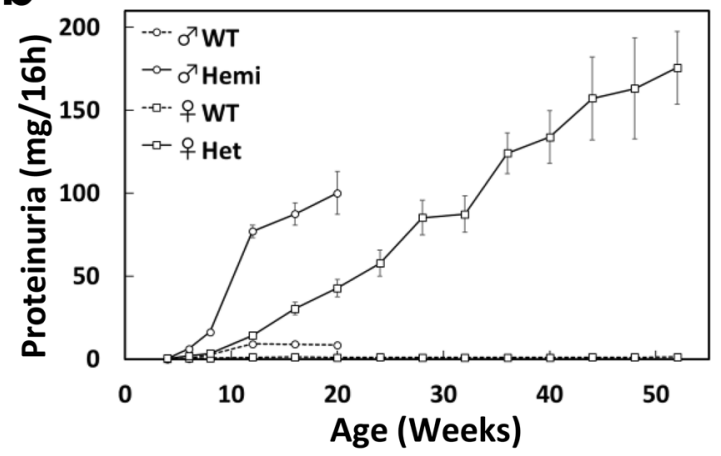

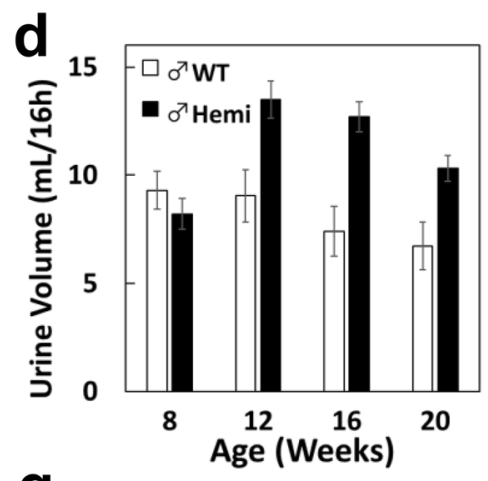

g

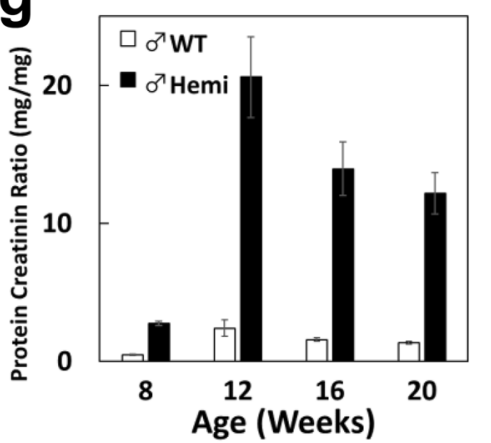

e

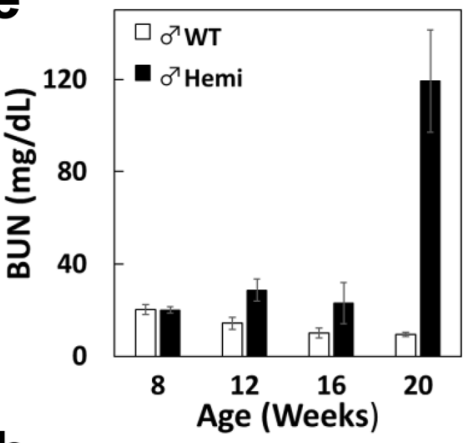

h

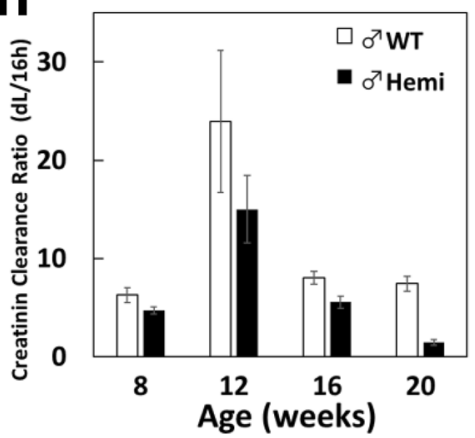

Figure 1. Physiological analyses in Col4a5 mutant rats. (a) Estimated survival functions in wildtype (WT) and Col4a 5 mutant (Hemi; hemizygous males, het; heterozygous females) rats. (b) Proteinuria in wildtype and Col4a 5 deficient rats from 4 to 52 weeks of age. (c-f) Measurements of body weight (c), urine volume (d), blood urea nitrogen (BUN) of serum (e), serum creatinine (Cre) (f), protein creatinine ratio (g), and creatinine clearance ratio (h) in wildtype and Col4a5 mutant males from 8 to 20 weeks of age.

(Fig. 4a, Supplementary Fig. 6). In contrast, the expression level of fibronectin, a marker of pathological deposition of the extracellular matrix (ECM), was increased in 12 weeks of age of hemizygous mutant males (Fig. 4b). We then observed fibronectin expression in heterozygous mutant females at 12 weeks of age (Fig. 4b, Supplementary Fig. 7).

Next, it has previously been reported that human, dog, and mouse Alport kidneys showed the progressive deposition in the GBM such as laminins ${ }^{32,33}$, and the progressive induction in glomeruli such as profibrotic cytokine TGF $\beta 1^{32}$ and matrix metalloproteinase (MMPs) ${ }^{34}$. To characterize such possibilities in Alport model rats, we performed immunostaining and Western blotting of Col4a 5 deficient male kidneys for measuring the levels of these proteins. We first observed that an antibody to the laminin $\beta 2$ chain exhibited intense staining of the GBM in Col4a 5 deficient males from 8 weeks of age (Fig. 5a, Supplementary Fig. 8a). To explore whether the TGF $\beta$ pathway was affected in Col4a5 deficient rats, we examined levels of phospho-Smad 3 which are the most critical mediators in TGF $\beta$ signaling pathway by Western blotting. We found that phosphorylation levels were increased in mutant male kidneys in comparison with those in wildtype (Fig. $5 \mathrm{~d}$ ). We then observed the protein expression of TGF $\beta$ downstream mediator $\mathrm{CTGF}^{35}$ in Col4a 5 deficient rats. Immunostaining analyses also showed the level of CTGF was increased in the GBM of mutant males by 12 weeks of age (Fig. 5b, Supplementary Fig. 8b). Moreover, we observed that an antibody to the MMP3/10 revealed intense staining of the GBM in Col4a 5 deficient males by 12 weeks of age (Fig. 5c, Supplementary Fig. 8c). In addition, we also detected increased MMP12 levels by Western blotting (Fig. 5d).

In the recently report, osteopontin (OPN) and LDL receptor (LDLR) are highly expressed in renal tubules of Alport mice ${ }^{36}$. To investigate the expression of OPN and LDLR in Alport model rats, we performed 

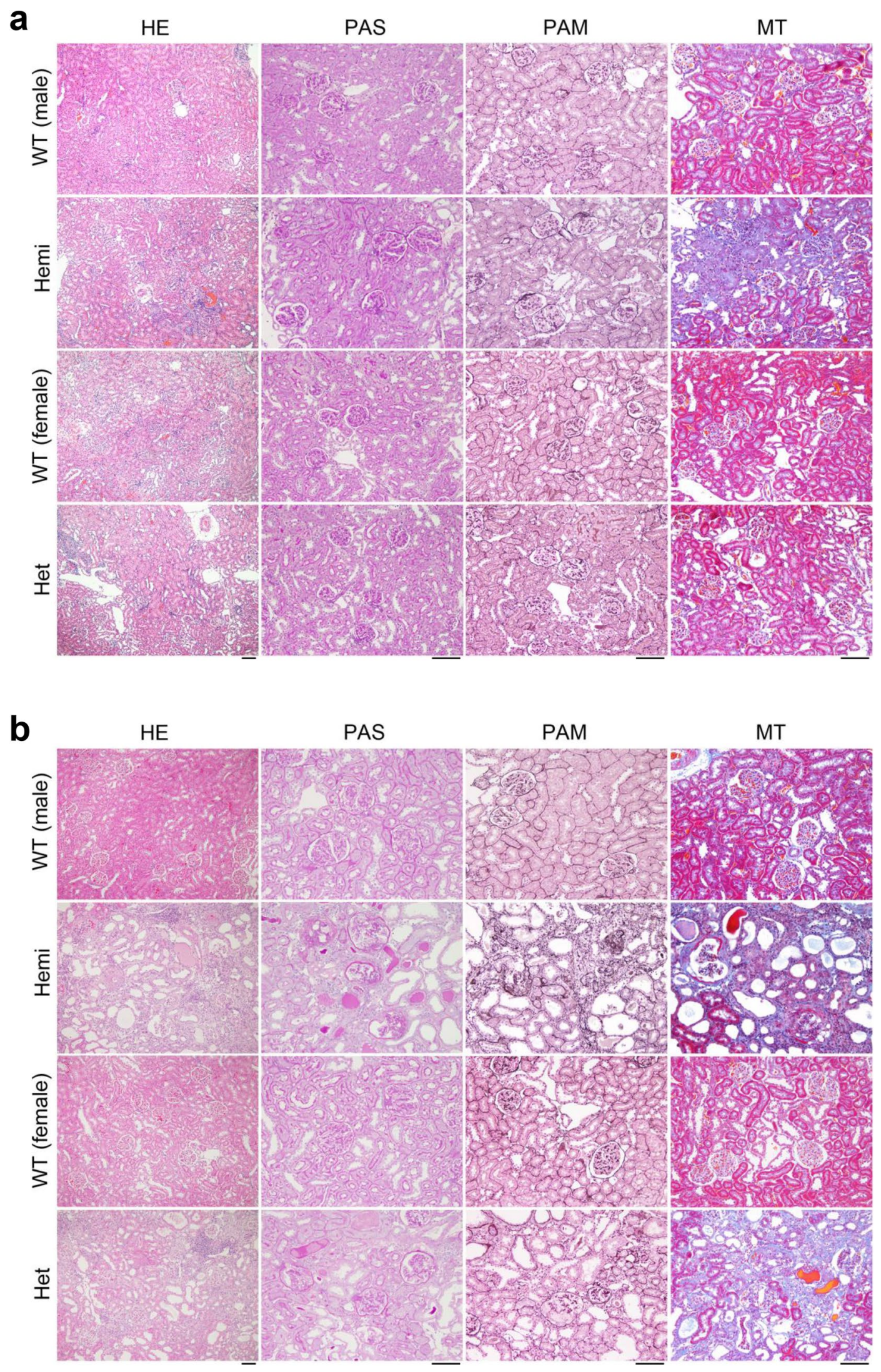

Figure 2. Histological analyses of Col4a5 deficient kidneys. (a,b) Representative microscopic images in wildtype (WT) and Col4a5 mutant (Hemi; hemizygous males, Het; heterozygous females) rats at 8 weeks (a) and 20 weeks (b) of age. These tissue sections were prepared and stained with hematoxylin and eosin (HE), periodic acid Schiff (PAS), periodic acid methenamine silver (PAM), and Masson trichrome (MT). Scale bars, $100 \mu \mathrm{m}$. 


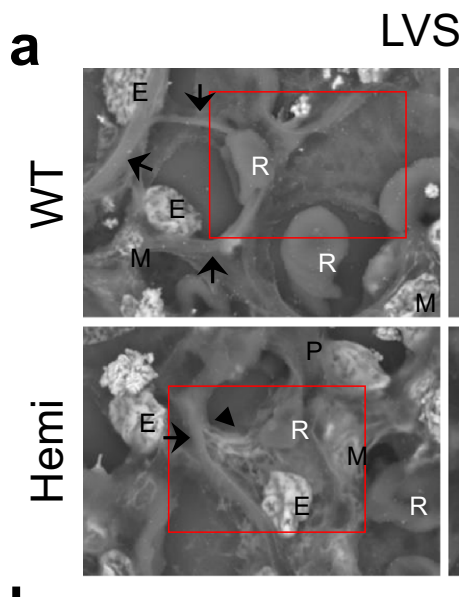

\section{SEM}

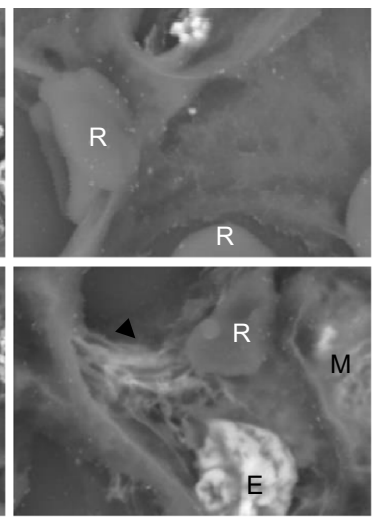

b
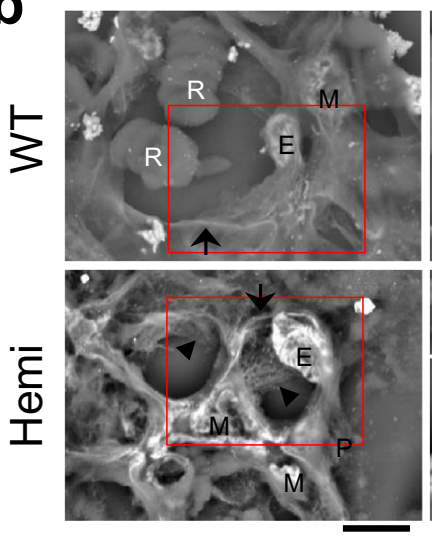
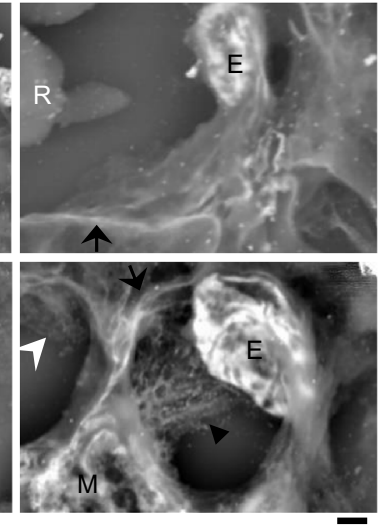
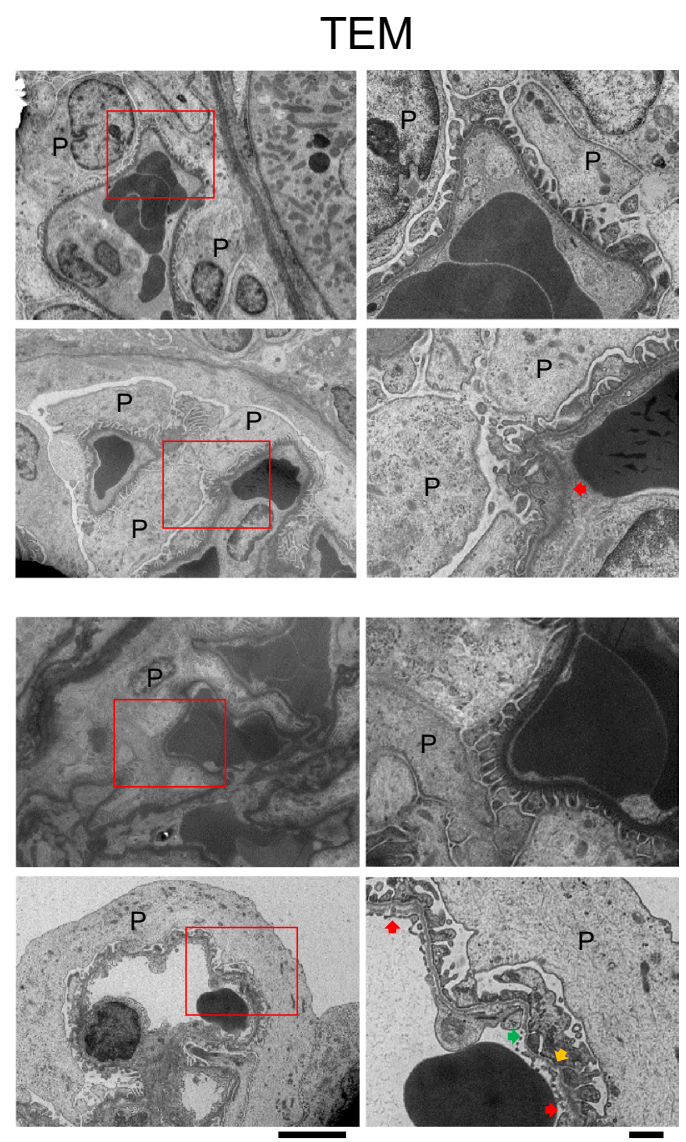

Figure 3. Electron photomicrographs of glomerular basement membranes in Col4a 5 mutant rats. (a,b) Representative low-vacuum scanning electron microscopy (LVSEM, left) and transmission electron microscopy (TEM; right) images in wildtype (WT) and Col4a 5 mutant (Hemi) males at 8 weeks (a) and 20 weeks (b) of age. Black and white arrowheads indicate the coarse meshwork structure of the GBM. Black arrows indicate cut side of the capillary walls. Red arrows indicate thickening of the GBM. Green arrows indicate thin patterns of the GBM. Yellow arrows indicate the splitting or fragmenting of the lamina densa. Red insets are revealed the higher magnification of left panels. E: Endothelial cells, M: Mesangial cells, P: Podocytes, R: Red blood cells. Scale bars, $5 \mu \mathrm{m}$ (left), $1 \mu \mathrm{m}$ (right).

immunostaining and Western blotting of Col4 $\alpha 5$ deficient male kidneys and/or plasma samples for measuring the levels of these proteins. Immunostaining analyses revealed the level of OPN was elevated in the renal tubules of mutant males from 8 weeks of age (Fig. 6a, Supplementary Fig. 9a). In addition, Western blotting also showed an increase of OPN expression in kidneys (Fig. 6c) and plasma (Fig. 6d) of Col4 $\alpha 5$ deficient males. In addition, LDLR expression was increased in the renal tubules of mutant males by 16 weeks of age by immunostaining (Fig. 6b, Supplementary Fig. 9b).

The expression of type IV collagen $\alpha 1-6$ in Col4 $\alpha 5$ deficient rats. The type IV collagen networks comprised of $\alpha 1 / \alpha 1 / \alpha 2$ (IV) (all basement membranes), $\alpha 3 / \alpha 4 / \alpha 5$ (IV) (GBM), and $\alpha 5 / \alpha 5 / \alpha 6$ (IV) (Bowman's capsule) protomers were observed in the glomeruli. To examine whether localizations and/or levels of the proteins were changed in Col4a 5 deficient rats, we investigated Col4a 5 deficient rats with graded levels of the other's type IV collagen $\alpha 1, \alpha 2, \alpha 3, \alpha 4$, and $\alpha 6$ (IV). First, we produced monoclonal antibodies that specifically recognize rat COL4A6 protein. We obtained rCol4A6 antibodies by injecting the antigen in Col4a5 deficient males, but we could not obtain any specific antibody injected to wildtype rats at all. Immunoblot analysis revealed that the monoclonal anti-rCOL4A6, reacted specifically with a band corresponding to the position of the similar molecular weights in recombinant rCOL4A6 proteins, and only reacted with rCOL4A6, but not with other rCOL4 proteins (Supplementary Fig. 10).

To determine whether localization of type IV collagens of a1-6 (IV) in the kidney of Col4a5 deficient rats changed, we performed the immunofluorescence analyses. At 8-20 weeks of age, COL4A5 expression was absent in hemizygous mutant males, and present in a mosaic pattern in heterozygous mutant females (Fig. 7a,b, Supplementary Fig. 11-14). The expressions of COL4A3, COL4A4, and COL4A6 were also absent in hemizygous mutant males, and present in a mosaic pattern in heterozygous mutant females (Fig. 7a,b, Supplementary Fig. 11-14). At 8 weeks of age, in contrast, expressions of COL4A1 and COL4A2 were present in Col $4 \alpha 5$ deficient rats (Fig. 7a, Supplementary Fig. 11). At 20 weeks of age, the kidney showed a strong accumulation of COL4A1 and COL4A2 
a
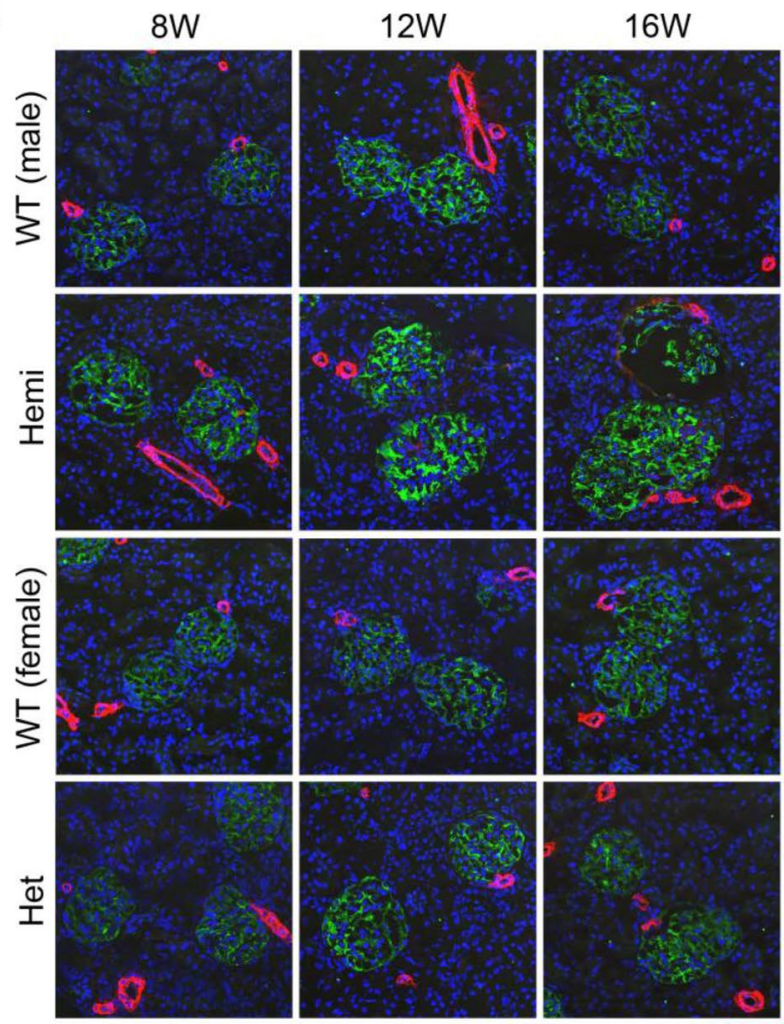

$\alpha$-SMA/Nestin/DAPI

b
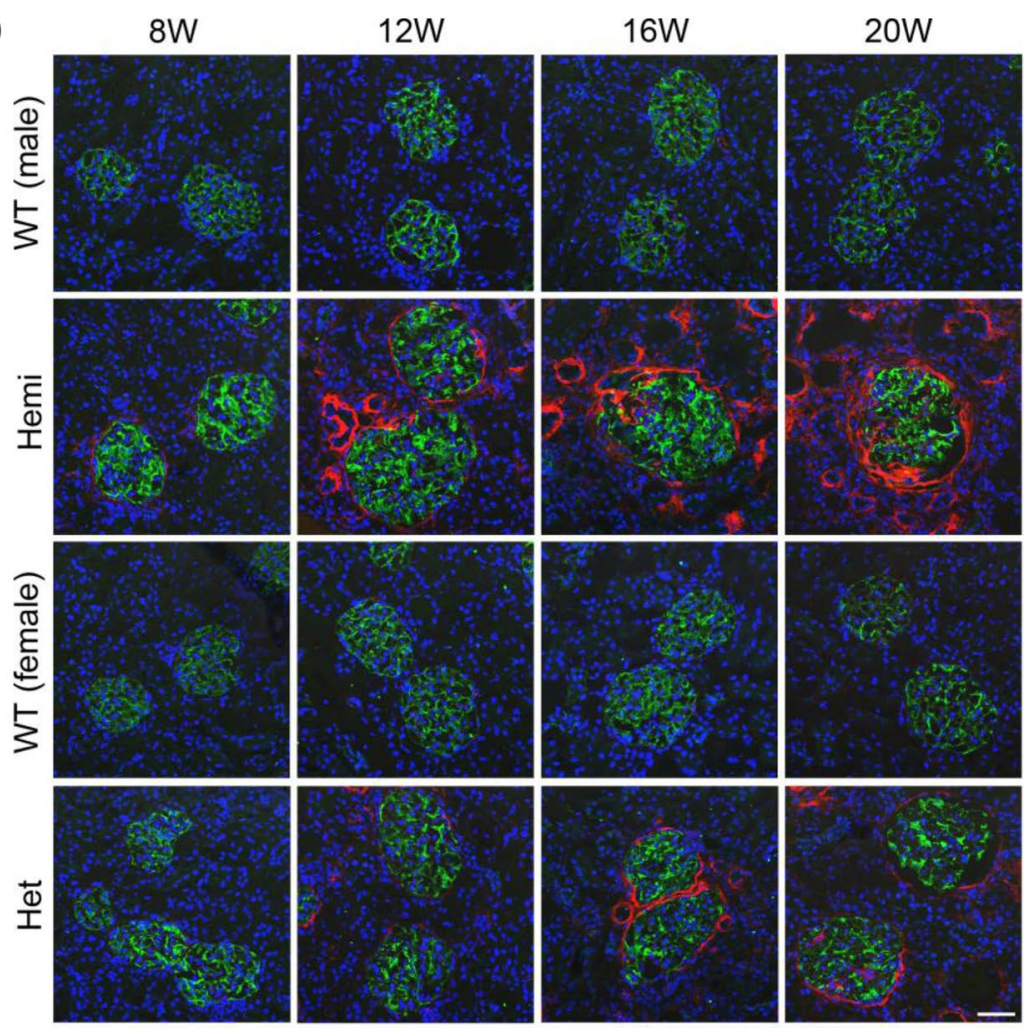

Fibronectin/Nestin/DAPI

Figure 4. Renal fibrosis in Col4a 5 deficient rats. (a,b) Immunostaining of kidney sections with a-SMA (a) or fibronectin (b) (red), nestin (green; glomeruli), and DAPI (blue; nuclei) in wildtype (WT) and Col4 $\alpha 5$ mutant (Hemi; hemizygous males, Het; heterozygous females) rats from 8 to 20 weeks of age. Scale bars, $50 \mu \mathrm{m}$. 

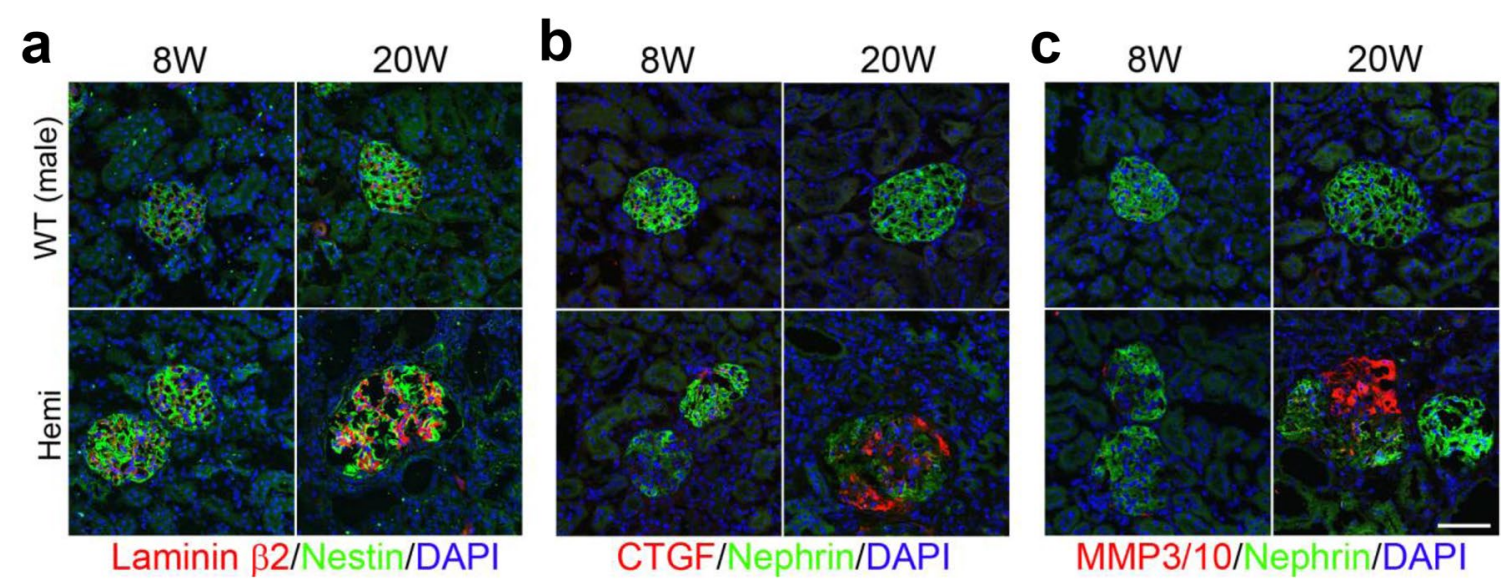

CTGF/Nephrin/DAPI

MMP3/10/Nephrin/DAPI

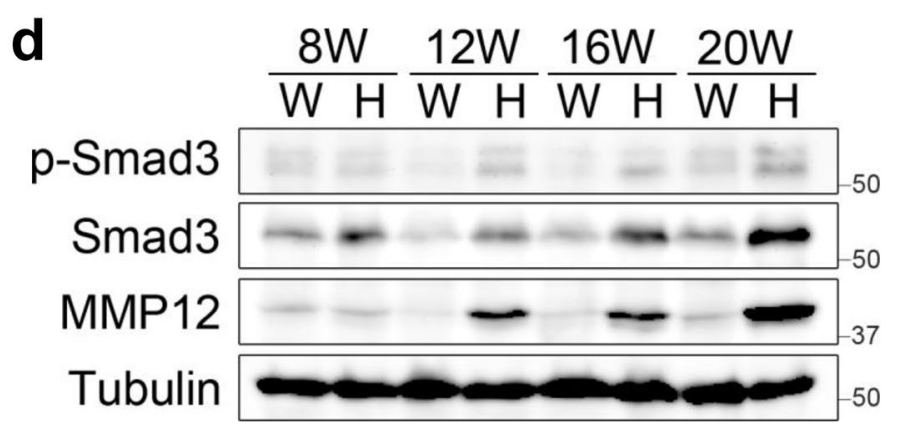

Figure 5. Immunostaining and Western blotting of the GBM in Col4a5 deficient kidneys. (a-c) Immunofluorescence analyses of rat kidney sections with antibodies against (a): Laminin $\beta 2$ (red), Nestin (green; glomeruli); (b): CTGF (red), Nephrin (green); (c): MMP3/10 (red), Nephrin (green); and DAPI (blue; nuclei) in wildtype (WT) and Col4 $\alpha 5$ mutant (Hemi) male rats at 8 weeks (left) and 20 weeks (right) of age. Scale bars, $50 \mu \mathrm{m}$. (d) Detection of phospho- and total Smad3, and MMP12 by Western blot analyses of the kidneys in wildtype (W) and Col4 $\alpha 5$ mutant $(\mathrm{H})$ male rats from 8, 12, 16, to 20 weeks of age. Tubulin was evaluated as an internal control.

proteins in both the GBM and Bowman's capsule (Fig. 7b, Supplementary Fig. 14). These data suggest that $\alpha 3 /$ $\alpha 4 / \alpha 5$ (IV) and $\alpha 5 / \alpha 5 / \alpha 6$ (IV) chains of type IV collagen disrupted in Col4 $\alpha 5$ deficient rats.

To verify the results of immunostaining, we analyzed COL4 protein expressions of noncollagenous domains 1 (NC1) by Western blot analyses that were prepared from kidneys of wildtype and hemizygous mutant males (Fig. 8). There were no bands detectable NC1 domains of type IV collagen $\alpha 3$, $\alpha 4$, and $\alpha 5$ (IV), derived from $\alpha 3 /$ a4/a5 (IV) complexes, in kidney of Col4 $\alpha 5$ deficient males (Fig. 8). Then, the NC1 domains of type IV collagen $\alpha 5$ and a6 (IV), derived from $a 5 / a 5 / a 6$ (IV) complexes, were also absent, whereas the blotting for the NC1 domains of type IV collagen $\alpha 1$ and $\alpha 2$ (IV), derived from $\alpha 1 / \alpha 1 / \alpha 2$ (IV) complexes, were present in hemizygous mutant males as well as wild type (Fig. 8). Therefore, these findings fully corroborate the results of immunofluorescence analyses.

\section{Discussion}

The present study showed that we successfully created a novel Alport syndrome rat model with the rGONAD technology. Col4a5 deficient rats revealed typical physiological, pathological, and also histological characteristics of Alport syndrome.

Occurrence of Alport syndrome is caused by mutations in COL4A3, COL4A4, or COL $4 A 5^{5}$. Several mouse models in Col4a3, Col4a 4, and Col4a 5 mutation have been known and characterized ${ }^{7-11,14}$. Col4a $3^{-/-}$or Col4a $4^{-/-}$ mice are well used for studying treatments of Alport syndrome compared to Col4a $5^{-/ Y}$ mice $^{35,37-39}$. Because, a survival ratio of Col4a $5^{-/ Y}$ mice was so diffusive ranged from 6 to 34 weeks ${ }^{10}$, in contrast, those of Col4a $3^{-/-}$or $\mathrm{Col} 4 \mathrm{a} 4^{-/-}$mice were more uniform (from 13 to 26 weeks) ${ }^{40,41}$. In this study, Col4a 5 mutant males died at 18 to 28 weeks (about $95 \%$ from 20 to 26 weeks) of age. Thus, it is useful to study experiments for prolong the lifespan of Col4a 5 deficient rats for effects of the drug, food, and other factors.

In the present study, we are able to produce Col4a5 mutant rats of WKY strain. Some number of glomeruli exhibited capillary tuft collapse at 8 weeks of age, and more glomeruli revealed abnormalities with crescent formation and focal sclerosis at the 20 weeks of age in Col4a 5 mutant males. The levels of BUN and Cre in serum highly increased in 20 weeks of age, and then died at around 24 weeks of age in hemizygous mutant males. These physiological and pathological features correspond to those of previously reported Col4a $3^{-/-}$with C57BL/6 mouse strain $^{13,15}$. However, Col4a $3^{-/-}$with $129 \mathrm{X} 1 / \mathrm{Sv}$ mouse strains showed much earlier progression of disease, died 

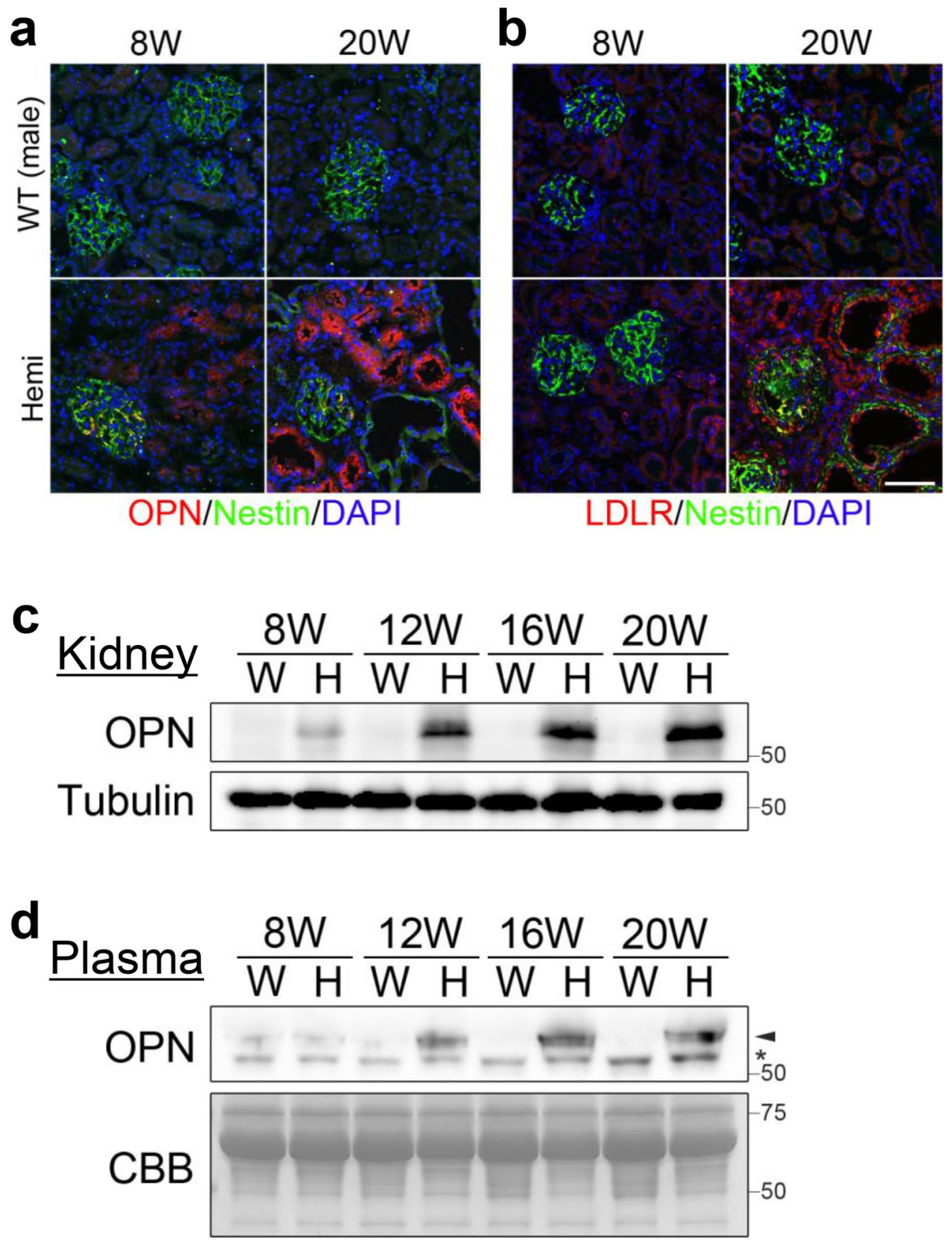

Figure 6. OPN and LDLR expressions in Col4a 5 deficient rats. (a,b) Immunostaining of kidney sections with (a) OPN or (b) LDLR (red), Nestin (green; glomeruli), and DAPI (blue; nuclei) in wildtype (WT) and Col4 $\alpha 5$ mutant (Hemi) male rats in wildtype (WT) and Col4 $\alpha 5$ mutant (Hemi) male rats at 8 weeks (left) and 20 weeks (right) of age. Scale bars, $50 \mu \mathrm{m}$. (c,d) Detection of OPN by Western blotting of the kidneys (c) or plasma (d) in wildtype (W) and Col4 $\alpha 5$ mutant $(\mathrm{H})$ male rats from $8,12,16$, to 20 weeks of age. Tubulin and CBB staining were evaluated as an internal control. Asterisk in (d) indicates non-specific signal.

at around 12 weeks of age $\mathrm{e}^{13,40,42}$. Therefore, comparison of a survival ratio between different strains of mice and rats should be carefully studied them, and further studies are required to draw a definite conclusion. In further study, other rat strains of Col4a5 mutant using the rGONAD method can be expected.

In the past decades, there were several Alport syndrome models such as mouse and $\operatorname{dog}^{16}$. Using this Alport model rat, there are certainly advantages compared with those of mouse and dog. Rat is cheaper than dog to breed and maintain, and much reliable than dog to design genetic study because of much number of pups and much shorter gestational age. On the other hand, rat is more readily than mouse to measure the proteinuria level. Then, rat provides enough glomeruli, blood, and urine to proceed experiments that require significant amounts of protein, facilitating proteomic approaches to understanding glomerular disease mechanisms compared with mouse. Therefore, we strongly suggest that Alport syndrome model rat with the rGONAD method can be useful in variety of experimentation for underlying the mechanism of renal diseases. 

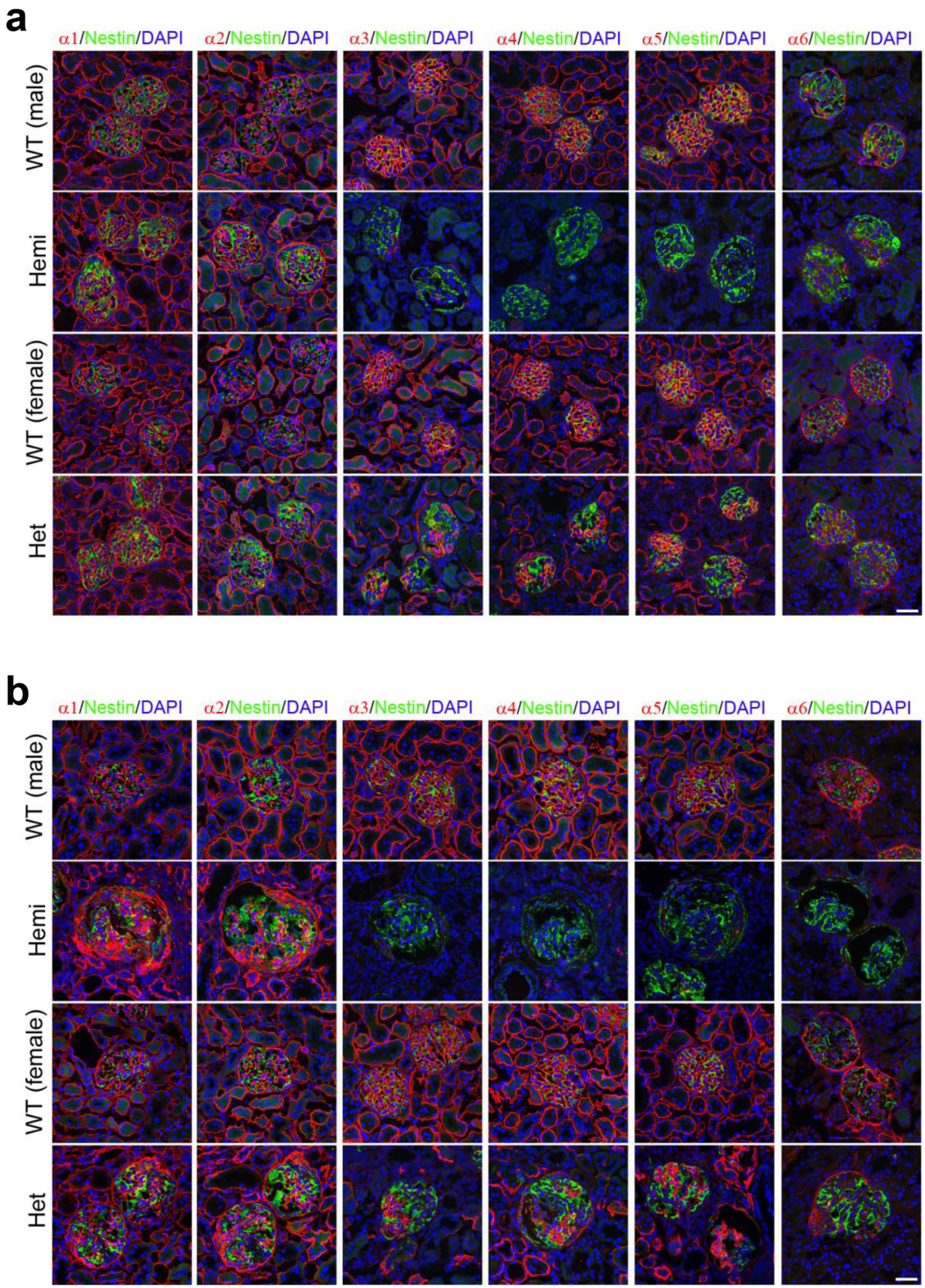

Figure 7. Type IV collagen distributions in Col4a 5 deficient kidneys. (a,b) Immunofluorescence analyses of kidney sections with antibodies against al-6 (IV) (red), nestin (green; glomeruli), and DAPI (blue; nuclei) in wildtype (WT) and Col4 $\alpha 5$ mutant (Hemi; hemizygous males, Het; heterozygous females) rats at 8 weeks (a) and 20 weeks (b) of age. Scale bars, $50 \mu \mathrm{m}$. 


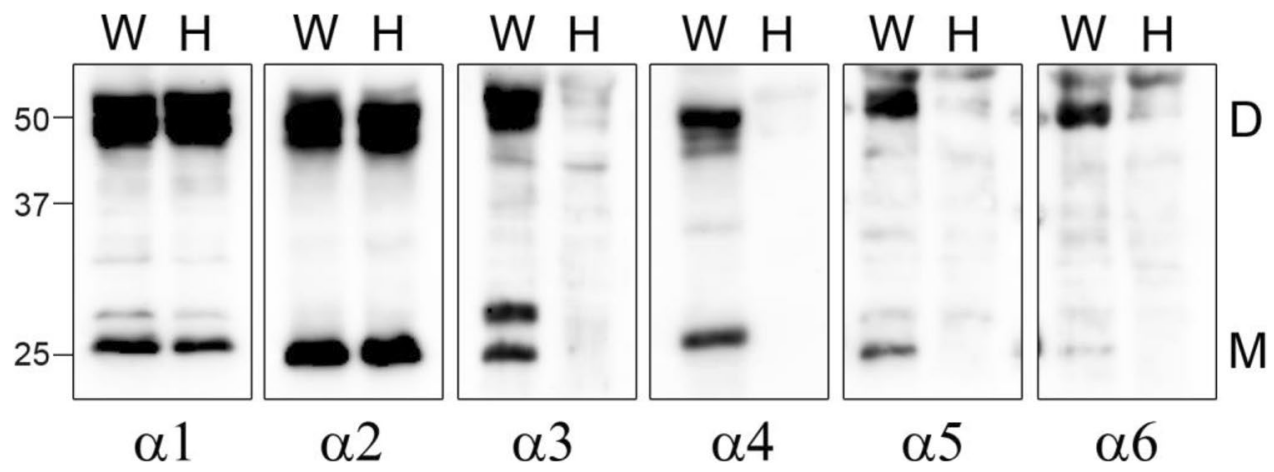

Figure 8. Western blot analyses of type IV collagen in Col4a 5 mutant kidneys. Collagenase-solubilized renal basement membranes from wildtype (W) and Col4a 5 mutant $(\mathrm{H})$ males at 8 weeks of age were separated by SDS-PAGE and blotted with antibodies that the specifically recognized $\alpha 1-6$ (IV) NC1 domains. D: NC1 dimer, M: NC1 monomer.

The laboratory rat has long been recognized as a preferred experimental animal in wide areas of biomedical science ${ }^{24,43}$. In some situation, rat is considered as a more relevant model among mammals. For example, physiology of rat is greatly well documented, because its larger body size affords the opportunity for serial blood draw studies. In particular, blood pressure measurement by telemetry is easier to perform and more reliable in rat compared to smaller mouse $\mathrm{e}^{44,45}$. Moreover, there are several rat strains in order to perform specific studies, i,e, SHR for hypertension ${ }^{46}$. Although genome engineering experiments of rat have yet not been extensively proceeded, it is readily applicable to produce the gene-editing mutant rats in Col4a5 gene of the strains by the rGONAD method. The model rat might provide new insight into the study of the relationship between renal disease such as Alport syndrome and other blood pressure related diseases.

In conclusion, we have described creation of a novel rat model of X-linked Alport syndrome. This rat model should be available for the study of progressive renal failure having basement membrane abnormality. Thus, Col4a 5 mutant rat is a reliable candidate for Alport syndrome model animal for underlying the mechanism of renal diseases and further identifying potential therapeutic targets for human renal diseases.

\section{Methods}

Animals. WKY/NCrl rats were obtained from Charles River. The rats were kept with a 12:12-h light: dark cycle. They were given free access of drinking water and food. All animals were handled in strict accordance with good animal practice as defined by the relevant national and/or local animal welfare bodies. All animal works were approved by the Shigei Medical Research Institute Animal Care Committee (permission number: $\# 17,006$ ), and were performed in accordance with relevant guidelines and regulations. The manuscript was prepared according to the ARRIVE guidelines.

Generation of type IV collagen $\alpha 5$ KO rats. Type IV collagen $\alpha 5$ deficient rats were produced by the rGONAD method as previously described ${ }^{28,30}$. Briefly, gene targeting strategy is designed to integrate tandem STOP codons into 27 bases after the first ATG in the rat Col4 $\alpha 5$ gene (Supplementary Fig. 1a). Guide RNAs were designed using CHOPCHOP (https://chopchop.cbu.uib.no/) (Supplementary Fig. la).

For the preparation of CRISPR/Cas9 reagents, Alt-R $\mathrm{R}^{\mathrm{mu}}$ CRISPR-Cas9 system (Integrated DNA Technologies [IDT, Coralville, IA]) was used in accordance with the manufacturer's protocol. Approximately $2-2.5 \mu$ of electroporation solution was injected into the oviductal lumen from up-stream of ampulla using a micropipette. The electroporation was performed using a NEPA21 (NEPAGENE Co. Ltd., Chiba, Japan).

Genotyping. Gene alteration was certified by PCR followed by DNA sequencing, as described previously ${ }^{47}$. Rat genomic DNA was isolated from ear-piece or tail. Genotyping was performed by PCR with the following primers (see also Supplementary Fig. 1A): rCol4a5-fw, (5'-GCTCTCTTCCCAATAACCCCT-3'), rCol4a5-rv, (5'-CAATTTTGACTTCCCTGGCCA-3').

Urine and blood parameters. Urine samples were collected for $16 \mathrm{~h}$ by placed rats in metabolic cages individually, every 4 weeks over 52 weeks (see Supplementary Table 1). Proteinuria level was measured by a modified method using $3 \%$ sulfosalicylic acid. Blood samples $(n=6$ each) were collected from the tail and centrifuged at $3000 \mathrm{~g}$ for $5 \mathrm{~min}$ to obtain blood serum. Blood urea nitrogen (BUN) of serum was measured using Colorimetric Detection Kit (Arbor Assays, Michigan, USA). Creatinine (Cre) levels of serum or urine were measured Jaffe's method (FUJIFILM Wako Pure Chemical Corporation, Osaka, Japan). Creatinine clearance ratio was calculated by (urine creatinine $[\mathrm{mg} / \mathrm{dl}]$ x urine volume $[\mathrm{dl}]$ )/serum creatinine $[\mathrm{mg} / \mathrm{dl}]$. All measurements were carried out according to manufacturer's recommended protocols. 
Histological analyses. Rat kidneys were soaked in $10 \%$ buffered neutral formalin at least overnight, and embedded in paraffin after dehydrated. The embedded kidneys were sliced 1-2 $\mu \mathrm{m}$ thickness. These slides were stained with hematoxylin and eosin (HE), periodic acid Schiff (PAS), periodic acid methenamine silver (PAM) and Masson's trichrome (MT) by standard methods.

Low vacuum scanning electron microscopy (LVSEM) and transmission electron microscopy (TEM). Under LVSEM, 3 dimensional ultrastructure of the GBM of Alport syndrome, the kidney was examined as described previously ${ }^{31}$. In brief, renal paraffin sections of $4 \mu \mathrm{m}$ thickness were stained with PAM. The sections on the slides were directly observed without a cover clip, with LVSEM (Hitachi TM4000; Hitachi Co. Ltd., Tokyo, Japan) at acceleration voltage of $15 \mathrm{kV}$ with $30 \mathrm{~Pa}$.

TEM was performed as described previously ${ }^{48}$. Ultrathin epoxy resin sections were prepared and observed using an H-7600 electron microscope (Hitachi Co. Ltd.).

Preparation of recombinant proteins. His-tagged (for production of antibody) and MBP-tagged (for immunoblotting) NC1 domains of type IV collagen a1-6 (V) were expressed in BL21-CodonPlus-RP (Agilent Technologies, Santa Clara, CA) transformed with pET-28a (Invitrogen) and pMAL (New England Biolabs, Beverly, MA), respectively. Each His or MBP fusion protein was purified through affinity chromatography with TALON metal affinity resin (Clonetech, Palo Alto, CA) or with amylose resin (New England Biolabs), respectively.

Antibodies. We produced rat monoclonal rCOL4A6, as described previously ${ }^{49,50}$. Briefly, the antigen emulsion was injected to Col4a5 deficient males. The treated rats were sacrificed 21 days after the injection, and the lymphocytes were fused with SP2/0-Ag14 myeloma cells. After the cell fusion, culture supernatants were screened to confirm positive clones by solid-phase enzyme-linked immunosorbent assay (ELISA).

The following primary antibodies were used: rat monoclonal type IV collagen a1(IV) (H11), a2 (IV) (H22), a3 (IV) (H31), a4 (IV) (H43), a5(IV) (H52) (In our institute) ${ }^{3}$; Tubulin (T9026), a-SMA (1A4) (Sigma, St. Louis, MO); phospho-Smad3 (ab52903), Fibronectin (ab6328), LDL receptor (ab52818), MMP12 (ab52897) (Abcam, Cambridge, UK); Smad3 (\#9523, Cell Signaling Technology, Beverly, MA); Nephrin (GP-N2, Progen, Germany); Nestin (66259-1-Ig, proteintech, Chicago, IL); Laminin $\beta 2$ (MAB2066), OPN (AF808) (R\&D systems Minneapolis, MN); CTGF (sc-373936), MMP3/10 (sc-374029) (Santa Cruz Biotechnology, Santa Cruz, CA); and MBP (New England Biolab). Primary antibodies were detected using species-specific secondary antibodies conjugated to either Alexa Fluor 488 or 555 (Molecular probes).

Tissue extract preparation and immunoblotting. Rat kidneys and blood plasma were homogenized directly in SDS-PAGE sample buffer. For collagenase-solubilized renal basement membranes, rat kidneys were homogenized and incubated at $37{ }^{\circ} \mathrm{C}$ for $4 \mathrm{~h}$ with $1 \mathrm{mg}$ kidney lysates and $200 \mu \mathrm{g}(1000 \mathrm{U})$ of collagenase (Brightase-C; Nippi, Okayama, Japan) in digestion buffer (20 mM HEPES, $10 \mathrm{mM} \mathrm{CaCl}$ ) with protease inhibitor cocktail (Nacalai, Kyoto, Japan). Protein concentrations for tissue extracts were determined by Coomassie Brilliant Blue staining by SDS-PAGE gels. The lysates were loaded, transferred, and subjected to Western blotting with specific antibodies as described previously ${ }^{51}$.

Immunofluorescence. Immunofluorescence analyses were examined, as described previously ${ }^{52}$. Briefly, rat kidneys were immersed in OCT compound, and snap-frozen in liquid nitrogen vapor. Subsequently, kidneys were sliced $5 \mu \mathrm{m}$ cryostat sections and placed on slides. After dehydration by acetone, blocked in $5 \%(\mathrm{v} / \mathrm{v})$ donkey serum for $30 \mathrm{~min}$ at room temperature. Sections were then incubated with primary antibodies overnight at $4{ }^{\circ} \mathrm{C}$ followed by PBS wash and incubated with appropriate secondary antibodies for $1 \mathrm{~h}$ at room temperature. DNA was also stained with $1 \mu \mathrm{g} / \mathrm{ml}$ DAPI. Fluorescence images were obtained by confocal microscopy (FV1200, Olympus, Japan).

\section{Data availability}

All data generated and analyzed during this study are included in this published article and its Supplementary Information.

Received: 18 May 2021; Accepted: 12 October 2021

Published online: 21 October 2021

\section{References}

1. Cosgrove, D. \& Liu, S. Collagen IV diseases: A focus on the glomerular basement membrane in Alport syndrome. Matrix Biol. 57-58, 45-54. https://doi.org/10.1016/j.matbio.2016.08.005 (2017).

2. Fidler, A. L., Boudko, S. P., Rokas, A. \& Hudson, B. G. The triple helix of collagens: An ancient protein structure that enabled animal multicellularity and tissue evolution. J. Cell Sci. https://doi.org/10.1242/jcs.203950 (2018).

3. Ninomiya, Y. et al. Differential expression of two basement membrane collagen genes, COL4A6 and COL4A5, demonstrated by immunofluorescence staining using peptide-specific monoclonal antibodies. J. Cell Biol. 130, 1219-1229. https://doi.org/10.1083/ jcb.130.5.1219 (1995).

4. Kruegel, J., Rubel, D. \& Gross, O. Alport syndrome-insights from basic and clinical research. Nat. Rev. Nephrol. 9, 170-178. https:// doi.org/10.1038/nrneph.2012.259 (2013).

5. Kashtan, C. E. et al. Alport syndrome: A unified classification of genetic disorders of collagen IV alpha345: A position paper of the Alport Syndrome Classification Working Group. Kidney Int. 93, 1045-1051. https://doi.org/10.1016/j.kint.2017.12.018 (2018). 
6. Bekheirnia, M. R. et al. Genotype-phenotype correlation in X-linked Alport syndrome. J. Am. Soc. Nephrol. 21, 876-883. https:// doi.org/10.1681/ASN.2009070784 (2010).

7. Cosgrove, D. et al. Collagen COL4A3 knockout: A mouse model for autosomal Alport syndrome. Genes Dev. 10, 2981-2992. https://doi.org/10.1101/gad.10.23.2981 (1996).

8. Miner, J. H. \& Sanes, J. R. Molecular and functional defects in kidneys of mice lacking collagen alpha 3(IV): Implications for Alport syndrome. J. Cell Biol. 135, 1403-1413. https://doi.org/10.1083/jcb.135.5.1403 (1996).

9. Korstanje, R. et al. A mouse Col4a4 mutation causing Alport glomerulosclerosis with abnormal collagen alpha3alpha4alpha5(IV) trimers. Kidney Int. 85, 1461-1468. https://doi.org/10.1038/ki.2013.493 (2014).

10. Rheault, M. N. et al. Mouse model of X-linked Alport syndrome. J. Am. Soc. Nephrol. 15, 1466-1474. https://doi.org/10.1097/01. asn.0000130562.90255.8f (2004).

11. Hashikami, K. et al. Establishment of X-linked Alport syndrome model mice with a Col4a5 R471X mutation. Biochem. Biophys. Rep. 17, 81-86. https://doi.org/10.1016/j.bbrep.2018.12.003 (2019).

12. Song, J. Y. et al. Bidirectional, non-necrotizing glomerular crescents are the critical pathology in X-linked Alport syndrome mouse model harboring nonsense mutation of human COL4A5. Sci. Rep. 10, 18891. https://doi.org/10.1038/s41598-020-76068-4 (2020).

13. Murata, T. et al. COL4A6 is dispensable for autosomal recessive Alport syndrome. Sci. Rep. 6, 29450. https://doi.org/10.1038/srep2 9450 (2016)

14. Falcone, S. et al. Modification of an aggressive model of Alport Syndrome reveals early differences in disease pathogenesis due to genetic background. Sci. Rep. 9, 20398. https://doi.org/10.1038/s41598-019-56837-6 (2019).

15. Kang, J. S. et al. Loss of alpha3/alpha4(IV) collagen from the glomerular basement membrane induces a strain-dependent isoform switch to alpha5alpha6(IV) collagen associated with longer renal survival in Col4a3-/- Alport mice. J. Am. Soc. Nephrol. 17, 1962-1969. https://doi.org/10.1681/ASN.2006020165 (2006).

16. Jansen, B. et al. Animal model of human disease: Hereditary nephritis in Samoyed dogs. Am. J. Pathol. 116, 175-178 (1984).

17. Atanur, S. S. et al. Genome sequencing reveals loci under artificial selection that underlie disease phenotypes in the laboratory rat. Cell 154, 691-703. https://doi.org/10.1016/j.cell.2013.06.040 (2013).

18. Mullins, L. J., Conway, B. R., Menzies, R. I., Denby, L. \& Mullins, J. J. Renal disease pathophysiology and treatment: Contributions from the rat. Dis. Model Mech. https://doi.org/10.1242/dmm.027276 (2016).

19. Yoshimi, K. \& Mashimo, T. Application of genome editing technologies in rats for human disease models. J. Hum Genet. https:// doi.org/10.1038/s10038-017-0346-2 (2017).

20. Aitman, T. J. et al. Copy number polymorphism in Fcgr3 predisposes to glomerulonephritis in rats and humans. Nature 439, 851-855. https://doi.org/10.1038/nature04489 (2006).

21. Sado, Y., Naito, I., Akita, M. \& Okigaki, T. Strain specific responses of inbred rats on the severity of experimental autoimmune glomerulonephritis. J. Clin. Lab. Immunol. 19, 193-199 (1986).

22. Sado, Y. et al. Isologous monoclonal antibodies can induce anti-GBM glomerulonephritis in rats. J. Pathol. 168, 221-227. https:// doi.org/10.1002/path.1711680211 (1992).

23. Sado, Y. et al. Induction of anti-GBM nephritis in rats by recombinant alpha 3(IV)NC1 and alpha 4(IV)NC1 of type IV collagen. Kidney Int. 53, 664-671. https://doi.org/10.1046/j.1523-1755.1998.00795.x (1998).

24. Meek, S., Mashimo, T. \& Burdon, T. From engineering to editing the rat genome. Mamm. Genome 28, 302-314. https://doi.org/ 10.1007/s00335-017-9705-8 (2017).

25. Szpirer, C. Rat models of human diseases and related phenotypes: A systematic inventory of the causative genes. J. Biomed. Sci. 27, 84. https://doi.org/10.1186/s12929-020-00673-8 (2020).

26. Takahashi, G. et al. GONAD: Genome-editing via Oviductal Nucleic Acids Delivery system: A novel microinjection independent genome engineering method in mice. Sci. Rep. 5, 11406. https://doi.org/10.1038/srep11406 (2015).

27. Ohtsuka, M. et al. i-GONAD: A robust method for in situ germline genome engineering using CRISPR nucleases. Genome Biol. 19, 25. https://doi.org/10.1186/s13059-018-1400-x (2018).

28. Kobayashi, T. et al. Successful production of genome-edited rats by the rGONAD method. BMC Biotechnol. 18, 19. https://doi. org/10.1186/s12896-018-0430-5 (2018).

29. Gurumurthy, C. B. et al. Creation of CRISPR-based germline-genome-engineered mice without ex vivo handling of zygotes by i-GONAD. Nat. Protoc. 14, 2452-2482. https://doi.org/10.1038/s41596-019-0187-x (2019).

30. Namba, M. et al. GONAD: A new method for germline genome-editing in mice and rats. Dev. Growth Differ. https://doi.org/10. $1111 /$ dgd.12746 (2021).

31. Okada, S. et al. Morphological diagnosis of Alport syndrome and thin basement membrane nephropathy by low vacuum scanning electron microscopy. Biomed. Res. 35, 345-350. https://doi.org/10.2220/biomedres.35.345 (2014).

32. Cosgrove, D. et al. Integrin alphalbetal and transforming growth factor-betal play distinct roles in alport glomerular pathogenesis and serve as dual targets for metabolic therapy. Am. J. Pathol. 157, 1649-1659. https://doi.org/10.1016/s0002-9440(10)64802-x (2000).

33. Kashtan, C. E. et al. Abnormal glomerular basement membrane laminins in murine, canine, and human Alport syndrome: Aberrant laminin alpha2 deposition is species independent. J. Am. Soc. Nephrol. 12, 252-260. https://doi.org/10.1681/ASN.V122252 (2001).

34. Meehan, D. T. et al. Biomechanical strain causes maladaptive gene regulation, contributing to Alport glomerular disease. Kidney Int. 76, 968-976. https://doi.org/10.1038/ki.2009.324 (2009).

35. Koepke, M. L. et al. Nephroprotective effect of the HMG-CoA-reductase inhibitor cerivastatin in a mouse model of progressive renal fibrosis in Alport syndrome. Nephrol. Dial. Transpl. 22, 1062-1069. https://doi.org/10.1093/ndt/gfl810 (2007).

36. Ding, W. et al. Osteopontin deficiency ameliorates Alport pathology by preventing tubular metabolic deficits. JCI Insight https:// doi.org/10.1172/jci.insight.94818 (2018).

37. Ninichuk, V. et al. Delayed chemokine receptor 1 blockade prolongs survival in collagen 4A3-deficient mice with Alport disease. J. Am. Soc. Nephrol. 16, 977-985. https://doi.org/10.1681/ASN.2004100871 (2005).

38. Cosgrove, D. et al. Lysyl oxidase like-2 contributes to renal fibrosis in Col4alpha3/Alport mice. Kidney Int. 94, 303-314. https:// doi.org/10.1016/j.kint.2018.02.024 (2018).

39. Omachi, K. et al. Metformin ameliorates the severity of experimental Alport syndrome. Sci. Rep. 11, 7053. https://doi.org/10.1038/ s41598-021-86109-1 (2021).

40. Andrews, K. L., Mudd, J. L., Li, C. \& Miner, J. H. Quantitative trait loci influence renal disease progression in a mouse model of Alport syndrome. Am. J. Pathol. 160, 721-730. https://doi.org/10.1016/S0002-9440(10)64892-4 (2002).

41. Gross, O. et al. Stem cell therapy for Alport syndrome: The hope beyond the hype. Nephrol. Dial. Transpl. 24, 731-734. https://doi. org/10.1093/ndt/gfn722 (2009).

42. Gross, O. et al. Preemptive ramipril therapy delays renal failure and reduces renal fibrosis in COL4A3-knockout mice with Alport syndrome. Kidney Int. 63, 438-446. https://doi.org/10.1046/j.1523-1755.2003.00779.x (2003).

43. Smith, J. R., Bolton, E. R. \& Dwinell, M. R. The rat: A model used in biomedical research. Methods Mol. Biol. 2018, 1-41. https:// doi.org/10.1007/978-1-4939-9581-3_1 (2019).

44. Jacob, H. J. The rat: A model used in biomedical research. Methods Mol. Biol. 597, 1-11. https://doi.org/10.1007/978-1-60327-3893_1 (2010). 
45. Aitman, T., Dhillon, P. \& Geurts, A. M. A RATional choice for translational research?. Dis. Model Mech. 9, 1069-1072. https://doi. org/10.1242/dmm.027706 (2016).

46. Hunziker, M. H., Saldana, R. L. \& Neuringer, A. Behavioral variability in SHR and WKY rats as a function of rearing environment and reinforcement contingency. J. Exp. Anal. Behav. 65, 129-144. https://doi.org/10.1901/jeab.1996.65-129 (1996).

47. Matsuyama, M., Nomori, A., Nakakuni, K., Shimono, A. \& Fukushima, M. Secreted Frizzled-related protein 1 (Sfrp1) regulates the progression of renal fibrosis in a mouse model of obstructive nephropathy. J. Biol. Chem. 289, 31526-31533. https://doi.org/ 10.1074/jbc.M114.584565 (2014).

48. Shimada, S. et al. Pathophysiological and molecular mechanisms involved in renal congestion in a novel rat model. Sci. Rep. 8, 16808. https://doi.org/10.1038/s41598-018-35162-4 (2018).

49. Kishiro, Y., Kagawa, M., Naito, I. \& Sado, Y. A novel method of preparing rat-monoclonal antibody-producing hybridomas by using rat medial iliac lymph node cells. Cell Struct. Funct. 20, 151-156. https://doi.org/10.1247/csf.20.151 (1995).

50. Sado, Y., Inoue, S., Tomono, Y. \& Omori, H. Lymphocytes from enlarged iliac lymph nodes as fusion partners for the production of monoclonal antibodies after a single tail base immunization attempt. Acta Histochem. Cytochem. 39, 89-94. https://doi.org/10. 1267/ahc.06001 (2006)

51. Matsuyama, M. et al. Defect of mitotic vimentin phosphorylation causes microophthalmia and cataract via aneuploidy and senescence in lens epithelial cells. J. Biol. Chem. 288, 35626-35635. https://doi.org/10.1074/jbc.M113.514737 (2013).

52. Koyano, T. et al. The p21 dependent G2 arrest of the cell cycle in epithelial tubular cells links to the early stage of renal fibrosis. Sci. Rep. 9, 12059. https://doi.org/10.1038/s41598-019-48557-8 (2019).

\section{Acknowledgements}

We are grateful to Chieko Takahashi for animal breeding, Dr. Yoshikazu Sado and Biomedical Research Core of Tohoku University Graduate School of Medicine for technical assistance, and Dr. Tohru Okigaki for critical comments on the manuscript. We would like to thank Dr. Fumihiro Shigei, Chairman of the Board, for financial support and continuous encouragements. This work was also supported in part by the Ryobi Teien Memory Foundation and Wesco Scientific Promotion Foundation.

\section{Author contributions}

M.N., T.K., M.K., T.K., M.F., and M.M. conceived and designed the experiments, M.N., T.K., M.K., T.K., T.H., and M.M. performed experiments, M.N. and M.M. wrote the manuscript. All authors read and approved the final manuscript.

\section{Competing interests}

The authors declare no competing interests.

\section{Additional information}

Supplementary Information The online version contains supplementary material available at https://doi.org/ 10.1038/s41598-021-00354-y.

Correspondence and requests for materials should be addressed to M.M.

Reprints and permissions information is available at www.nature.com/reprints.

Publisher's note Springer Nature remains neutral with regard to jurisdictional claims in published maps and institutional affiliations.

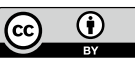

Open Access This article is licensed under a Creative Commons Attribution 4.0 International License, which permits use, sharing, adaptation, distribution and reproduction in any medium or format, as long as you give appropriate credit to the original author(s) and the source, provide a link to the Creative Commons licence, and indicate if changes were made. The images or other third party material in this article are included in the article's Creative Commons licence, unless indicated otherwise in a credit line to the material. If material is not included in the article's Creative Commons licence and your intended use is not permitted by statutory regulation or exceeds the permitted use, you will need to obtain permission directly from the copyright holder. To view a copy of this licence, visit http://creativecommons.org/licenses/by/4.0/.

(C) The Author(s) 2021, corrected publication 2021 